\title{
Estratégias de educação nutricional na prevenção e controle da obesidade infantil
}

\author{
Nutritional education strategies in the prevention and control of childhood obesity
}

Estrategias de educación nutricional en la prevención y control de la obesidad infantil

Waleska Gualberto da Silva ${ }^{1 *}$, Walnara Arnaud Moura Formiga1', Rafael Ferreira Lima², Izabela Letícia de Andrade e Silva ${ }^{1}$, Lourena de Melo Almeida ${ }^{1}$, Gislane de Almeida Linhares ${ }^{1}$, Thiago Alves Viana $^{1}$, Jessilânia Gomes da Silva ${ }^{1}$, Sabrina Bezerra da Silva ${ }^{1}$, Érika Maria Gomes de Araújo Nóbrega ${ }^{3}$.

\section{RESUMO}

Objetivos: O presente estudo teve como objetivo revisar e investigar as estratégias de educação nutricional na prevenção e controle da obesidade infantil. Métodos: Trata-se de uma revisão de literatura, onde utilizou-se de artigo, livros e material publicados nos últimos 10 anos, publicados nas bases de dados Biblioteca Virtual de Saúde, Scielo e Google Acadêmico. Resultados: A Educação Alimentar e Nutricional é uma importante ferramenta de prevenção e controle da Obesidade Infantil, e a Escola é entendida como local ideal para implementação das metodologias pedagógicas a serem trabalhadas. Considerações Finais: O estudo mostrou a importância de se trabalhar educação nutricional nas escolas, para que essas práticas sejam aplicadas no cotidiano nas escolhas dos alimentos, refletindo a curto prazo em crianças menos propensas a obesidade infantil e a longo prazo pessoas mais saudáveis, partindo do pressuposto que se identificam quais fatores positivos e negativos da sua alimentação por meio da Educação nutricional.

Palavras-chave: Educação nutricional, Obesidade infantil, Estratégias.

\section{ABSTRACT}

Objectives: This study aimed to review and investigate nutritional education strategies in the prevention and control of childhood obesity. Method: This is a literature review, where it used articles, books and material published in the last 10 years, published in the Virtual Health Library, SciELO and Google Scholar databases. Results: Food and Nutrition Education is an important tool for the prevention and control of Childhood Obesity, and the School is understood as an ideal place to implement the pedagogical methodologies to be worked on. Final considerations: The study showed the importance of working with nutritional education in schools, so that these practices are applied in everyday life in food choices, reflecting in the short term in children less prone to childhood obesity and in the long term healthier people, based on the assumption that identify which positive and negative factors of their diet through nutritional education.

Key words: Nutritional education, Child obesity, Strategies.

\section{RESUMEN}

Objetivos: Este estudio tuvo como objetivo revisar y investigar estrategias de educación nutricional en la prevención y control de la obesidad infantil. Métodos: Esta es una revisión de la literatura, donde se utilizaron artículos, libros y material publicado en los últimos 10 años, publicado en las bases de datos de la Biblioteca Virtual en Salud, SciELO y Google Scholar. Resultados: La educación alimentaria y nutricional es una herramienta importante para la prevención y el control de la obesidad infantil, y la escuela se entiende como un lugar ideal para implementar las metodologías pedagógicas en las que se trabajará. Consideraciones Finales: El estudio mostró la importancia de trabajar con educación nutricional en las escuelas, de modo que estas prácticas se apliquen en la vida cotidiana en la elección de alimentos, reflejando a corto plazo en los niños menos propensos a la obesidad infantil y en las personas más saludables a largo plazo, basándose en el supuesto de que Identificar qué factores positivos y negativos de su dieta a través de la educación nutricional.

Palabras clave: Educación nutricional, Obesidad infantil, Estrategias.

\footnotetext{
${ }^{1}$ Centro Universitário de Patos (UNIFIP), Patos - PB. *E-mail: waleska.gual@gmail.com

2Universidade Federal de Campina Grande (UFCG), Pombal - PB.

3Universidade Federal da Paraíba (UFPB), João Pessoa - PB.
} 


\section{INTRODUÇÃO}

É notório o aumento na prevalência de sobrepeso no passar dos anos, assim como também quando se trata da obesidade, associados se tornam o quinto maior fator de risco para mortalidade no mundo. Esses valores são observados em países desenvolvidos e em desenvolvimento, sendo que em populações menos favorecidas é duas vezes maior, todavia, em números absolutos, os países em desenvolvimento possui quatro vezes mais crianças com sobrepeso ou obesidade quando comparados às nações desenvolvidas. Estima-se que até o atual ano, 2020, as estatísticas de sobrepeso e obesidade na população mundial para crianças de até 5 anos seja de até $9,1 \%$ variando até $14,1 \%$ em países desenvolvidos a $8,6 \%$ nos países em desenvolvimento (ONIS M, et al., 2010).

Com elevada prevalência do sobrepeso e obesidade, surge a preocupação com o aparecimento de doenças crônicas não-transmissíveis (DCNT), dentre essas a hipertensão, diabetes mellitos, assim como também, esteatose hepática e transtornos psicossociais resultantes da estigmatização social. Esses índices tornam-se importantes quanto observados na população infantil, e com isso é essencial a criação de políticas públicas voltadas para a prevenção da obesidade e a redução dos índices da obesidade na população pediátrica (LIRA AR, et al., 2010; SILVEIRA JAC, et al., 2011).

O âmbito escolar é o ideal para a implementação dessas políticas, visto que a maioria das crianças passa grande parte do tempo na escola, ademais, o ambiente escolar tem influência sobre a saúde, pois as escolas fornecem aos estudantes as ferramentas necessárias para que eles entendam as orientações de saúde divulgadas pelos diversos meios de comunicação. A escola ainda exerce o papel crucial para 0 desenvolvimento psicológico e emocional das crianças, e pode incluir as informações mais atualizadas sobre saúde no currículo tradicional ou em disciplinas específicas voltadas para a promoção de saúde (SILVEIRA JAC, et al., 2011).

Isto posto, parece acertado dizer que a educação alimentar e nutricional visa a reeducação dos hábitos alimentares, e está vinculada à produção de informações que sirvam como subsídio para auxiliar na tomada de decisão dos indivíduos. Atividades em grupo ou individuais desenvolvidas em escolas são fundamentais na perspectiva da promoção de hábitos saudáveis (KOVACS C, et al., 2010.).

Visto isso, o presente artigo teve como objetivo revisar e investigar as estratégias de Educação Nutricional na prevenção e controle da obesidade infantil por meio de uma revisão de literatura.

\section{REVISÃO BIBLIOGRÁFICA}

\section{Globalização, mudanças culturais e sociais: reflexo na cultura alimentar}

Desde o início da Revolução Industrial, em meados do século XVIII, a estrutura organizacional das sociedades vem sendo modificada progressivamente ao longo dos tempos. À medida que os acontecimentos de entorno econômico se moldavam grandes impactos eram gerados ao estilo de vida das pessoas. Afetando a maneira como produziam seus alimentos, deixando a economia de subsistência e consequentemente os campos, gerando o êxodo rural.

De acordo com Vesentini JW (2015), tais mudanças que construíram a ideia dos Estados Nacionais, fomentou a regionalização da Indústria e consequentemente gerou destaque de algumas dessas regiões de produção, o que repercutiu de forma significativa posteriormente em meados dos anos 1970, com uma nova fase do capitalismo e da sociedade moderna, a Terceira Revolução Industrial, ou Revolução Técnicocientífica, e a Globalização, nas quais algumas nações específicas se sobressaíram e consequentemente conseguiram influenciar ou modular vários setores econômicos e sociais das demais populações (COLLYER FRS, 2015).

Com a Globalização, houve uma maior unificação mundial de povos, economias, tornando-os mais interligados. O termo globalização começou a ser usado após a década de 70 como referência desta interdependência a que se submetiam os povos e economias da superfície terrestre, porém pode ser considerado um processo antigo, que já davam seus indícios nos séculos $\mathrm{XV}$ e XVI, com as Grandes Navegações e expansões do Capitalismo Mercantilista (VESENTINI JW, 2015). 
A repercussão deste processo rápido de mudanças no mundo, que suscita controvérsias, levou a uma série de modificações em diversas áreas da vida humana, tais como: a reorganização de estruturas seculares, como a família, a inserção da mulher no mercado de trabalho, surgimento da Internet e redes sociais, valorizando relações à distância, video games, tablets, minicomputadores e smartphones, além de aumento de oferecimento e de armazenagem de alimentos sazonais, agora disponíveis facilmente nos centros urbanos, em forma de enlatados, envasados, mini ou ultraprocessados, e várias outras maneiras de conservação conseguidas pela Indústria moderna que oferecem comodidade e praticidade aos consumidores atuais, cada vez mais sem tempo, surgindo por causa disso também novas tendências alimentares como fast food, junke food e a atual slow food (SCINTA W, 2013; SILVA ACB, et al., 2015).

Levando em conta ainda as mudanças na conformação da estrutura social das últimas décadas, houve uma expansão no número de indivíduos que realizam suas refeições fora de casa, consumindo principais refeições como almoço e jantar em restaurantes e lanchonetes (SANTOS MV, et al., 2011).

Frente a isso, houve drástico impacto nos valores alimentares das grandes populações mundiais que se tornaram cada vez mais influenciadas a adotarem padrões alimentares que vão de encontro com características alimentares particulares de seu povo e que em sua maioria não se configuram de qualidade do ponto de vista nutricional, oferecendo gêneros ou preparações ricos em açúcar, sódio e gorduras, o que tem gerado um fator de risco sério para a saúde em seus consumidores, a obesidade. De certo modo, a indústria alimentícia é um esclarecedor exemplo de como a organização capitalista modifica hábitos alimentares culturais e finda por minimizar a cultura alimentar de um povo (SILVA ACB, et al., 2015).

\section{Obesidade: desafio mundial}

A obesidade é o acúmulo de gordura corporal excessiva, com prejuízo á saúde, decorrente de vários fatores sejam esses genéticos ou ambientais, como padrões dietéticos e de atividade física ou ainda fatores individuais de suscetibilidade biológica, entre muitos outros, que interagem na etiologia da patologia (ASSOCIAÇÃO BRASILEIRA PARA O ESTUDO DA OBESIDADE E SÍNDROME METABÓLICA, 2016).

Caracterizada ainda como uma condição complexa de dimensões sociais, biológicas e psicossociais consideráveis, podendo eventualmente afetar qualquer pessoa, de qualquer idade ou grupo socioeconômico, em qualquer lugar do mundo, sendo mais frequente nas classes menos favorecidas, responsável pela causa de inúmeras consequências como alterações no aparelho cardiovascular, sistema pulmonar, aparelho gastrintestinal e aparelho gênito-urinário e reprodutor, pode causar também complicações metabólicas e outras alterações (COSTA MJC, et al., 2013).

Nesta realidade, a obesidade é hoje considerada uma epidemia do século XXI, ela é encarada como a maior desordem nutricional nos países ocidentais. A Organização Mundial de Saúde (OMS) classifica a obesidade como o maior problema de saúde pública que a sociedade, dos nossos dias, enfrenta. Mas esta epidemia não afeta apenas os países desenvolvidos, tem-se registrado um crescimento significativo de pessoas com excesso de peso e obesidade em países em desenvolvimento (ASSOCIAÇÃO BRASILEIRA PARA O ESTUDO DA OBESIDADE E SÍNDROME METABÓLICA, 2016).

A prevalência de obesidade e sobrepeso nas Américas (62\% em adultos com mais de 20 anos de idade) é a maior de todas as regiões da OMS. A obesidade e o sobrepeso afetam cerca de 7 em cada 10 adultos no Chile, nos Estados Unidos e no México. Da mesma forma, as taxas de prevalência têm aumentado continuamente em crianças e adolescentes e os dados disponíveis indicam que $20 \%$ a $25 \%$ têm sobrepeso ou obesidade (ORGANIZAÇÃO PAN-AMERICANA DA SAÚDE - ORGANIZAÇÃO MUNDIAL DE SAÚDE, 2014).

Segundo dados de Ezzati M, et al. (2016), que mostram um estudo onde foram avaliadas as tendências no índice de massa corporal de adultos em 200 países de 1975 a 2014, com aproximadamente 19, 2 milhões de participantes e colaboração de inúmeros pesquisadores de cada país, é possível detectar que existem mais pessoas com sobrepeso e obesidade que desnutridas no mundo. Destacando ainda, que se as tendências pós-2000 continuarem, a probabilidade de se cumprir o objetivo global de obesidade, que é a meta global de conter a obesidade mundial em 2025 proposta pela Organização Mundial de Saúde (OMS), é 
praticamente zero. Ao contrário, se essas tendências continuarem, em 2025, a prevalência de obesidade global atingirá $18 \%$ em homens e deve ultrapassar os $21 \%$ em mulheres; obesidade grave irá atingir $6 \%$ dos homens e $9 \%$ das mulheres. No entanto, a prevalência de pessoas abaixo do peso continua nas regiões mais pobres do mundo, especialmente no sul da Ásia.

A Pesquisa de Orçamentos Familiares (POF) (2008-2009), realizada em parceria entre o IBGE e o Ministério da Saúde, analisando dados de 188 mil pessoas brasileiras em todas as idades, mostrou que a obesidade e o excesso de peso têm aumentado rapidamente nos últimos anos, em todas as faixas etárias. Neste levantamento, $50 \%$ dos homens e $48 \%$ das mulheres se encontram com excesso de peso, sendo que $12,5 \%$ dos homens e 16,9\% das mulheres apresentam obesidade (BRASIL, 2016).

A obesidade em adultos é determinada pelo Índice de Massa Corporal (IMC) que é calculado dividindo-se o peso (em $\mathrm{Kg}$ ) pelo quadrado da altura (em metros). O resultado revela se o peso está dentro da faixa ideal, abaixo ou acima do desejado. O resultado é dado em $\mathrm{Kg} / \mathrm{m}^{2} \mathrm{com}$ classificação de: de 30 a 34,9 obesidade grau I (leve), de 35 a 39,9 obesidade grau II (moderada), e acima de 40 obesidade grau III (mórbida) (OMS, 1998).

Em crianças são usadas as curvas com classificação em percentis ou score $-Z$, sendo que os valores de percentil maiores ou iguais a 97 e valores de score-z maior ou igual +2 indicadores de obesidade em crianças menores de 10 anos, e adolescentes valores de percentil acima do percentil 99,9 e escore-z maior que +3 (ORGANIZAÇÃO MUNDIAL DE SAÚDE, 2007; BRASIL-SISVAN, 2008).

\section{Obesidade infantil: suas causas e consequências}

Uma vez que estes acontecimentos históricos favoreceram a mudança e a modulação dos aspectos socioeconômicos e culturais que afetam em cheio aqueles relacionados à vida e alimentação dos indivíduos tornando-os obesos, com isso o número de crianças que se enquadram nessas características, excesso de peso corporal, aumentou também significativamente nos últimos anos, tendo vários fatores como contribuintes (MOREIRA MSF, et al., 2014).

Segundo a POF (2009), o número de crianças de 5 a 9 anos com excesso de peso (sobrepeso ou obesidade) era bastante significativo $(33,5 \%$ da amostra). Na pesquisa, foi possível detectar também que houve maior aquisição de produtos industrializados nos domicílios pesquisados, independente da classe social, e são indicativos dessas mudanças nos padrões alimentares, levando ao ganho de peso.

Apesar da dieta brasileira apresentar gêneros alimentícios tidos como marcadores saudáveis na alimentação tradicional, como feijão, arroz, tapioca e outros desenvolvidos do encontro de suas três matizes étnicas (indígena, europeia e africana) criando uma rica cultura alimentar, houve um aumento no consumo de alimentos processados e ultraprocessados, ricos em açúcar, sódio (refrigerantes, refrescos industrializados, biscoitos recheados e salgadinhos) e pouca ingestão de frutas, verduras e hortaliças que divergem com 0 Guia Alimentar para a População Brasileira e as recomendações da OMS (SILVA ACB, et al., 2015).

Junto a isso, surge o sedentarismo, devido a transição tecnológica que tem supervalorizado os aparelhos eletrônicos como ferramenta de diversão e rede de relacionamentos, em detrimento das brincadeiras populares regionais que valorizavam os costumes e práticas de um povo e estimulava a criação de vínculos afetivos entre aqueles que praticavam tais atividades recreacionais.

Além disso, com o crescimento das cidades e centros urbanos, aumentou-se a violência pública num modo geral, o que só contribuiu para que atividades que pudessem ser realizadas dentro de casa, sem muita exposição nas ruas, fossem ganhando espaço e confiança de pais e responsáveis pelas crianças, o que propiciou o comportamento sedentário mundial deste século (DIEZ-GARCIA RW, CERVATO-MANCUSO AM, 2013; RODRIGUES-CIACCHI EM, CAMPOS SH, 2013; VIEIRA MNC, 2013).

Com a prática de consumir mais refeições fora do lar, outros hábitos alimentares foram sendo incorporados às famílias, como substituição das preparações da refeição por lanches, sanduíches, hambúrgueres, salgadinhos, refrigerantes, o que só favorece a instalação da obesidade tanto em adultos como em crianças (RODRIGUES AGM, et al., 2013). 
Com a implantação de mercado globalizado, muitas lanchonetes multinacionais e franquias alimentares, acabaram por se instalar no Brasil e para atrair seus consumidores utilizam-se de várias manobras propagandistas muito atraentes para as crianças. Através da oferta de combos alimentares com brinquedos exclusivos como brinde, embalagens de produtos alimentares nos supermercados muito coloridos, bem acabadas e sofisticadas que causam grande desejo de consumo (SILVA ACB, et al., 2015).

Dessa maneira muitas são as vias atuais, no Brasil e no mundo, que viabilizam a instalação de um quadro de obesidade nas crianças desde tenra idade. O problema, porém, não se trata apenas da presença ou não da obesidade, mas sua correlação com geração de outras doenças, já que a obesidade infantil é fator de risco potencial para numerosas doenças que antes afetavam apenas adultos obesos, tais como hipertensão arterial, diabetes, apneia obstrutiva do sono e hipercolesterolemia (SCINTA W, 2013; MOREIRA MSF, et al., 2014; SILVA ACB, et al., 2015).

\section{Estratégias de educação nutricional como prevenção e controle da obesidade infantil: uma resposta eficiente}

Considera-se que o complexo quadro de morbimortalidade brasileiro em sua relação com a alimentação (marcado pela coexistência de Doenças Crônicas Não Transmissíveis (DCNTs), obesidade, anemia, carências de micronutrientes, fome e desnutrição) e a baixa frequência de atividade física, só podem ser enfrentados por ações que impactem de forma integrada todas essas dimensões do problema (BRASIL, 2014a).

Promover a alimentação adequada e saudável requer desenvolver ações voltadas à promoção da saúde, nutrição e alimentação da população; à garantia da qualidade nutricional e segurança dos alimentos; à produção do conhecimento e o acesso à informação; e à implementação de políticas públicas e estratégias sustentáveis e participativas de produção, comercialização e consumo de alimentos, respeitando as múltiplas características culturais do país. Portanto, implica repensar políticas públicas que possam incidir sobre o comportamento e o consumo alimentar da população brasileira, bem como a promoção da atividade física (CONSELHO NACIONAL DE SEGURANÇA ALIMENTAR E NUTRICIONAL, 2007).

Com base nessas informações, foi lançado em 2014 o documento da Estratégia Intersetorial de Prevenção e Controle da Obesidade: "orientando sobre modos de vida e alimentação adequada e saudável para a população brasileira". Nele são eleitos seis eixos de ação para efetiva e eficaz prevenção e controle da obesidade. Destacam-se, contudo, aquelas estratégias que focam na promoção e educação para formação de hábitos e práticas alimentares saudáveis: Ações de Educação, comunicação e informação e a Promoção de modos de vidas saudáveis nos ambientes/territórios, configurando-se os Eixo de Ação 2 e 3 , respectivamente. O papel dos processos educativos e informacionais na vida e na construção de valores políticos e sociais dos indivíduos, fazendo com que a educação seja vista como uma ferramenta importante para estimular o pensamento crítico e permitir o exercício da autonomia e do autocuidado (BRASIL, 2014b).

Tal conhecimento, concebido aqui como um conjunto dinâmico de saberes e fazeres acumulados e socializados ao longo da história de cada pessoa, resulta das informações percebidas sobre a realidade que o cerca, o que inclui fatos e memórias de seu processamento e das ações que obedecem às estratégias definidas pela vontade (D'AMBROSIO U, 2011).

A associação entre obesidade, práticas alimentares e conhecimentos de nutrição foi avaliada por Triches e Giuliani em 573 escolares matriculados em escolas municipais de um município do Rio Grande do Sul. Os autores observaram que a obesidade se mostra associada com menor nível de conhecimento de nutrição e práticas alimentares menos saudáveis, acrescentando que crianças com essas características apresentam cinco vezes mais chances de serem obesas (DETREGIACHI CR e BRAGA TMS, 2011).

Faz-se necessário, portanto, que a realização desta educação transformadora que insere a educação em saúde, bem como a nutricional e alimentar saia de uma abordagem apenas científica, do ponto de vista das ciências naturais ou biológicas, mas que se expandam às demais áreas de conhecimento. Saia de uma abordagem sanitarista, muito usada anteriormente como meio preventivo de doenças, e vá ao encontro das realidades existenciais dos indivíduos despertando-os à capacidade de se fazer parte do processo e 
conhecedor não só de um resultado físico ou situacional, mas de todos os possíveis fatores que desencadeiam tais emblemas de saúde ou nutrição (SANTOS LA, 2012).

A EAN é uma das principais estratégias para a promoção da alimentação adequada e saudável e pressupõe processos articulados e permanentes de problematização, reorganização de valores, atitudes e geração de autonomia, diferentemente das estratégias de informação e comunicação. Para organizar e estruturar este tema, em novembro de 2012, foi lançado o Marco Referencial da Educação Alimentar e Nutricional para as Políticas Públicas, o qual deverá apoiar a atuação de diversos atores no âmbito do espaço público. (BRASIL, 2012a).

A escola é o local ideal para a implementação dessas estratégias porque a maioria das crianças passa grande parte do tempo na escola. Além disso, o ambiente escolar tem influência sobre a saúde, já que fornecem aos estudantes as ferramentas necessárias para que eles entendam as orientações de saúde divulgadas pelos diversos meios de comunicação. Exercendo um papel fundamental no desenvolvimento psicológico e emocional das crianças, podendo incluir as informações mais atualizadas sobre saúde no currículo tradicional ou em disciplinas específicas (como educação física ou nutricional) voltadas para a promoção da saúde (RAMOS FP, et al., 2013).

Desde 1977, o Conselho Federal de Educação tinha destacado a importância da saúde ser considerada um dos Temas Transversais dos Parâmetros Curriculares Nacionais, (PCNs), ao indicar que os Programas de Saúde não deveriam ser encarados como matéria ou disciplina, mas como um tema a fazer parte do processo formador, intimamente ligado a missão da escola e podendo ser abordado em transversal com os variados componentes curriculares, particularmente o campo das Ciências, os estudos Sociaias e a Educação Física (MEC-SEF, 1998).

O Programa Saúde na Escola (PSE), política intersetorial da Saúde e da Educação que foi instituído em 2007, por sua vez, consegue fazer esta ponte, permitindo que por meio das políticas de saúde e educação voltadas às crianças, adolescentes, jovens e adultos da educação pública brasileira houvesse de forma comum a promoção à saúde e educação integral (BRASIL, 2007).

Soma-se a ele, o Programa Nacional de Alimentação Escolar (PNAE) que é uma das políticas públicas voltados à segurança alimentar e nutricional mais bem estabelecida e respeitada no país e no mundo, abrange todo o território nacional visando a garantia de uma alimentação no ambiente escolar mais saudável e adequada aos receptores do programa. Destacando na Lei 11.947 de 2009 e a Resolução no 26, de 17 de junho de 2013 , ambas no seu art. $2^{\circ}$, no qual dispõe como uma das diretrizes da Alimentação Escolar: "II - a inclusão da educação alimentar e nutricional no processo de ensino e aprendizagem, que perpassa pelo currículo escolar, abordando o tema alimentação e nutrição e o desenvolvimento de práticas saudáveis de vida na perspectiva da segurança alimentar e nutricional;" (BRASIL, 2013).

Assim Guerra PH, et al. (2016), ao estudar intervenções e desenvolvidas no ambiente escolar com fins na prevenção e/ou redução do excesso de peso consideram que a presença dos pais/responsáveis e a comunidade no processo, além de que período de intervenção mais longo (superior ou igual a 6 meses) foram mais exitórios.

No âmbito escolar, há uma predominate lacuna de outras experiências exitórias que disponham estratégias teórico-metodológicas para dar suporte as práticas de EAN. Fazendo com que fatores importantes na formação dos hábitos aliementares e inciados na infância sejam olhados com mais atenção afim de construir tais estratégias, ao observar questões fisiológicas e psicosocioculturais, além das econômicas que podem propiciar o acesso a alimentos, educação de qualidade (SANTOS LA, 2012; RAMOS FP, et al., 2013).

Avalia-se, nesse contexto, o papel da Escola como ambiente gerador de hábitos, dentre eles os alimentares que permitem ao aluno sair de sua intimidade e valores alimentares recebidos no seio familiar para comungar de hábitos construídos de forma coletiva pela sociedade ou grupo onde está se inserindo. Assim, ao favorecer a prova de novos alimentos, preparações, ter acesso a informações nutricionais, discutir sobre tabus, mitos, e novas descobertas dietéticas e implementação da Educação Nutrional a Escola se torna importante ferramenta de construção social e política, formando cidadões mais conscientes e saudáveis (RAMOS FP, et al., 2013). 


\section{CONSIDERAÇÕES FINAIS}

Com base nos achados parece que a EAN é ferramenta valiosa no processo de prevenção e combate a Obesidade Infantil, e a escola é ambiente mais que favorável para este processo. No entanto, embora seja nítida esta percepção, e hajam número pertinente de estudos que trazem algumas destas intervenções, não há uma consonância sobre quais estratégias teórico-metodológicos merecem mais destaque ou aprimoramento, há muito que se averiguar sobre a subjetividade que é dada ao professor e à escola para conduzir tais temas. Nesse contexto, é essecial a inserção do nutricionista no Programa Saúde na Escola (PSE) e Programa Nacional de Alimentação Escolar (PNAE), para que hábitos saudáveis quanto a alimentação sejam exercidos e assim haja redução da prevelência de sobrepeso e obesidade na fase infantil e adulta, visto que as escolas alimentares são tomadas a partir da infância.

\section{REFERÊNCIAS}

1. ASSOCIAÇÃO BRASILEIRA PARA O ESTUDO DA OBESIDADE E SÍNDROME METABÓLICA (ABESO). Mapa da Obesidade. Disponível em:<http://www.abeso.org.br/atitude-saudavel/mapa-obesidade $>$.

2. BRASIL, Fundo Nacional de Desenvolvimento da Educação. Programa Nacional de Alimentação Escolar: Dados sobre o programa. Maio, 2016.

3. MINISTERIO DA SAÚDE. Ministério da Educação. Orientações sobre o Programa Saúde na Escola para a elaboração dos Projetos Locais. Brasília, 2007.

4. MINISTÉRIO DA EDUCAÇÃO. Fundo Nacional de Desenvolvimento da Educação. Resolução no 26, de 17 de junho de 2013. Dispõe sobre o atendimento da alimentação escolar aos alunos da educação básica no âmbito do Programa Nacional de Alimentação Escolar - PNAE.

5. MINISTÉRIO DA SAÚDE. Sistema de Vigilância Alimentar e Nutricional. Protocolos do Sistema de Vigilância Alimentar e Nutricional - SISVAN na assistência à saúde. Brasília, DF, 2008.

6. INSTITUTO BRASILEIRO DE GEOGRAFIA ESTATÍSTICA. Pesquisa do Orçamento Familiar. 2008-2009.

7. MINISTÉRIO DO DESENVOLVIMENTO SOCIAL E COMBATE À FOME. Câmara Interministerial de Segurança Alimentar e Nutricional. Estratégia Intersetorial de Prevenção e Controle da Obesidade: Recomendações para Estados e Municípios. Maio 2014a.

8. MINISTÉRIO DO DESENVOLVIMENTO SOCIAL E COMBATE À FOME. Câmara Interministerial de Segurança Alimentar e Nutricional. Estratégia Intersetorial de Prevenção e Controle da Obesidade: Promovendo modos de vida e Alimentação Adequada e Saudável para a população Brasileira. Setembro 2014b.

9. COLLYER FRS. Revolução Industrial: aspectos políticos e sociais da maior revolução da idade moderna. Revista Jus Navigandi. 2015; 1(4242).

10. CONSELHO NACIONAL DE SEGURANÇA ALIMENTAR E NUTRICIONAL. Relatório Final- GT: "Alimentação Adequada e Saudável". Brasília, DF. 26 fev 2007.

11. COSTA MJC, et al. Desafios no Tratamento da Obesidade - Pesquisas Recentes e Diferentes Abordagens Nutricionais, Psicológicas e Físicas. p. 143-209. In: COSTA, M. J. C. [Org]. Nutrição Clínica: Uso do Sistema de Equivalentes na Prática Dietoterápica. 2 ed, João Pessoa, Editora da UFPB, 2013.

12. D'AMBROSIO U. Formação de valores um enfoque transdisciplinar In: MOLL, J. Caminhos da Educação Integral no Brasil: Direito a outros tempos e espaços Educativos. Artes Médicas, 2011.

13. DETREACHI CR, BRAGA TMS. Projeto "criança saudável, educação dez": resultados com e sem intervenção do nutricionista. Revista de Nutrição. 2011; 24(1): 51-59.

14. DIEZ-GARCIA RW, CERVATO-MANCUSO AM. Mudanças Alimentares e Educação Nutricional. Rio de janeiro: Guanabara-Koogan, [reimpr] 2013.

15. EZZATI M, et al. Trends in adult body-mass index in 200 countries from 1975 to 2014: a pooled analysis of 1698 population-based measurement studies with 19.2 million participants. Revista The Lancet. 2016; 387(10026): 1377 1396.

16. GUERRA PH, et al. Physical activity, and nutrition education at the school environment aimed at preventing childhood obesity: evidence from systematic reviews. J Pediatr, Rio de Janeiro, 2016;92:15-23.

17. KOVACS C, et al. Aplicação de uma ferramenta de educação nutricional em grupo para pacientes com excesso de peso e sua efetividade na mudança de parâmetros antropométricos. Rev Socidade de Cardiologia do Estado de São Paulo. 2010; 20(1): 9-13.

18. LIRA AR, et al. Hepatic steatosis in a school population of overweight and obese adolescente. J Pediatr. 2010; 86(1): 45-52.

19. MEC, Ministério da Educação. Secretaria de Educação Fundamental. Parâmetros Curriculares Nacionais: apresentação dos Temas Transversais e Ética. Brasília, DF, 1998.

20. MOREIRA MSF, et al. Doenças associadas à obesidade infantil. Revista Odontológica de Araçatuba. 2014; 35(1): 60-66.

21. ORGANIZAÇÃO MUNDIAL DE SAÚDE. Development of a WHO growth reference for school-aged children and adolescents. Bulletin of the World Health Organization. vol. 85 n. 9, 2007. 
22. ORGANIZAÇÃO PAN-AMERICANA DA SAÚDE- ORGANIZAÇÃO MUNDIAL DE SAÚDE. Plano de Ação para Prevenção da Obesidade em Crianças e Adolescentes. 53ํㅡㄹ Conselho Diretor, 66a Sessão do Comitê Regional da OMS para as Américas. Washington, D.C., EUA, 29 de set-3 de out de 2014. Washington: OPAS; 2014.

23. ONIS M, BLOSSNER M, BORHGI E. Global prevalence and trends of overweight and obesity among preschool children. Am J Clin Nutr. 2010; 92(1): 1257-64.

24. RAMOS FP, et al. Educação alimentar e nutricional em escolares: uma revisão de literatura. Rio de Janeiro. Cadernos de Saúde Pública. 2013; 29(11): 2147-2167.

25. RODRIGUES AGM, et al. Perfil da escolha alimentar de arroz e feijão na alimentação fora de casa em restaurante de bufê por peso. Ciências \& Saúde Coletiva. 2013; 18(2): 335-346.

26. RODRIGUES-CIACCHI EM, CAMPOS SH. Programa de Educação Nutricional com Grupo de Adolescentes: Problematização como Estratégia para análise e Mudanças do Comportamento Alimentar. In: DIEZ-GARCIA RW, CERVATO-MANCUSO AM. Mudanças Alimentares e Educação Nutricional. Rio de janeiro: Guanabara-Koogan, [reimpr] 2013 p. 297-308.

27. SANTOS LA. O fazer educação alimentar e nutricional: algumas contribuições para reflexão. Revista Ciências \& Saúde Coletiva. 2012; 17(2): 453-462.

28. SANTOS MV, et al. Os Restaurantes por peso no contexto de alimentação saudável fora de casa. Revista de Nutrição. 2011; 24(4): 641-649.

29. SCINTA W. Bounce: A Weight-Loss Doctor's Plan for a Happier, Healthier, and Slimmer Child. ed. 1, Fayetteville, Nova York, Medical Weight Loss of New York, PLLC, 234p., 2013.

30. SILVA ACB, et al. Educação alimentar e nutricional, cultura e subjetividades: a escola contribuindo para a formação de sujeitos críticos e criativos em torno da cultura alimentar. Demetra: Alimentação, Nutrição \& Saúde. 2015; 10(2): 247-257.

31. SILVEIRA JAC, et al. A efetividade de intervenções de educação nutricional nas escolas para prevenção e redução de ganho excessivo de peso em crianças e adolescentes: uma revisão sistemática. Jornal de Pediatria. 2011; 87(5): 382-92.

32. VESENTNI JW. Módulo: Economia e espaço geográfico. Espaço Geográfico e Industrialização, p. 4-12. In: SERIACOPI et al. Sistema de ensino ser: ensino médio. Caderno 2. Línguas: humanas: aluno. ed. 2, São Paulo, Editora Ática, 2015.

33. VIEIRA MNC, et al. Programa Nacional em grupo para Crianças e Adolescentes com Obesidade Ambulatorial In: DIEZ-GARCIA RW, CERVATO-MANCUSO AM. Mudanças Alimentares e Educação Nutricional. Rio de janeiro: Guanabara-Koogan, [reimpr] 2013 p. 297-308. 